\title{
Doença celíaca, gastrite atrófica autoimune e Helicobacter pylori na anemia refratária ao ferro oral
}

Ana Carolina Teixeira, ${ }^{1}$ Hugo Ribeiro, ${ }^{2}$ Helena Moreno, ${ }^{1}$ Cláudio Martins, ${ }^{1}$ Sandra Magano Silva, ${ }^{1}$ Alexandra Rafael, ${ }^{1}$ Daniel Canelas, ${ }^{2}$ Margarida Badior, ${ }^{3}$ Teresa Melo, ${ }^{3}$ Henrique Coelho ${ }^{3}$

\section{RESUMO}

Objetivos: Identificar o número de casos positivos para doença celíaca (DC), gastrite atrófica autoimune (GAAI) e infeção por Helicobacter pylori (Hp) em doentes com anemia ferropénica refratária ao ferro oral (ARF).

Tipo de estudo: Observacional longitudinal retrospetivo.

Local: Serviço de Hematologia do Centro Hospitalar Vila Nova de Gaia/Espinho

População: Doentes observados em primeira consulta por ARF entre 2011 e 2016.

Métodos: Recolha de dados através do processo clínico eletrónico (dados sociodemográficos, origem do pedido de consulta e dos métodos complementares de diagnóstico). Análise descritiva realizada no programa Microsoft Excel $201{ }^{\circledR}$.

Resultados: Identificaram-se 48 casos de ARF (9,3\% das anemias ferropénicas), dos quais metade efetuou rastreio completo com pesquisa de DC, GAAl e infeção por Hp, 19 doentes realizaram apenas para uma ou duas das entidades do estudo e cinco doentes não fizeram este rastreio. Em termos globais, e considerando apenas aqueles que fizeram despiste de pelo menos uma das entidades clínicas do estudo $(n=43)$, verifica-se que a prevalência de DC foi de aproximadamente 4,7\%, de GAAI de 14\% e a infeção por $\mathrm{Hp}$ de $30,2 \%$. Foi impossível avaliar a resposta à erradicação com $\mathrm{Hp}$, uma vez que dos 13 casos identificados esta foi desconhecida em seis casos. Dos sete casos conhecidos, o efeito da erradicação do Hp sobre a anemia foi resolutivo em apenas um doente.

Conclusão: Os resultados identificaram numa elevada percentagem de doentes com ARF, a existência de GAAI e infeção por HP, o que está de acordo com o referido em estudos internacionais, reforçando o papel destas entidades no desenvolvimento da ARF na população portuguesa. O estudo reforça a importância do despiste da DC, GAAl e infeção por Hp nos casos de ARF.

Palavras-chave: Anemia ferropénica; Doença celíaca; Gastrite atrófica; Helicobacter pylori; Anemia refratária.

\section{INTRODUÇÃO}

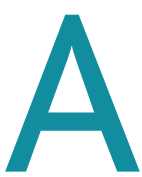
anemia é um problema de saúde pública a nível mundial com grandes repercussões na qualidade de vida e com uma prevalência global de $32,9 \%,{ }^{1}$ sendo a deficiência de ferro a causa mais frequente. ${ }^{2-5}$

A anemia por deficiência de ferro pode resultar da ingestão inadequada, diminuição da absorção, aumento das necessidades e/ou do aumento das perdas de fer-

1. USF Arco do Prado

2. USF Barão do Corvo

3. Centro Hospitalar Vila Nova de Gaia/Espinho ro. ${ }^{2,5}$ Por outro lado, a diminuição da absorção de ferro pode ser devida a condições cirúrgicas e médicas. Os distúrbios que afetam o trato gastrointestinal, por exemplo, a gastrite atrófica autoimune (GAAI), a infeção por Helicobacter pylori (Hp) e a doença celíaca (DC), prejudicam a absorção de ferro e levam à resistência ao tratamento com ferro oral. Pode ainda ocorrer redução funcional da absorção de ferro secundária ao aumento de hepcidina e à degradação de ferroportina. ${ }^{3-4}$ Além disso, alterações hereditárias em genes que codificam proteínas que regulam a absorção do ferro podem ser causa de anemia e de refratariedade à terapêutica com 
ferro, de que é exemplo a Iron refractory iron deficiency anemia (IRIDA). ${ }^{4}$

Estima-se que, em Portugal, 20\% dos adultos apresentem anemia e que $15 \%$ tenham ferropenia. ${ }^{6} \mathrm{~A}$ investigação etiológica é fundamental, sendo que nos homens e nas mulheres pós-menopáusicas a realização de estudo endoscópico digestivo é consensual.

No entanto, frequentemente não se identifica a etiologia da anemia e muitos destes casos não respondem à suplementação com ferro oral. Este facto tem atraído a atenção para a DC, GAAI e infeção por Hp como possíveis causas de anemia ferropénica refratária ao ferro oral (ARF), tendo como mecanismo fisiopatológico de base a diminuição da capacidade de absorção do ferro, como mencionado. Atualmente, em Portugal apenas o rastreio de DC está recomendado pela Direção-Geral da Saúde. ${ }^{7}$

A prevalência da refratariedade ao ferro oral é difícil de definir sendo muitas vezes afetada pela compliance do doente e pela experiência do clínico. ${ }^{3}$ Em Portugal não encontrámos qualquer estudo que avalie a influência da DC, GAAI e da infeção pelo Hp na ARF.

O objetivo principal deste estudo foi identificar, entre os doentes com ARF referenciados para uma unidade de hematologia de um centro hospitalar da região Norte de Portugal, o número de casos positivos para DC, GAAI e infeção por Hp. Como objetivo secundário, procurou-se avaliar a evolução da anemia após a erradicação do Hp.

\section{MÉTODOS}

Tratou-se de um estudo observacional longitudinal retrospetivo.

A população da amostra foram todos os utentes observados em primeira consulta de hematologia no Centro Hospitalar Vila Nova de Gaia/Espinho, por ARF, no período de 1 de janeiro de 2011 a 31 de dezembro de 2016. Em 2011 foi implementado um protocolo no serviço de despiste de Hp, GAAI e DC na anemia ferropénica sem causa aparente, que se manteve em aplicação no período temporal deste estudo.

Critérios de inclusão: doentes com anemia ferropénica (definida como hemoglobina sérica $<12 \mathrm{~g} / \mathrm{dL}$ nas mulheres $\mathrm{e}<13 \mathrm{~g} / \mathrm{dL}$ nos homens, com volume globular médio < 80fl e presença de ferropenia (ferritina $<30 \mathrm{ng} / \mathrm{dL})^{4}$ e sem resposta ao ferro oral (suplementa- ção mínima de 100mg de ferro elementar/dia, sem aumento de pelo menos $1 \mathrm{~g} / \mathrm{dL}$ de hemoglobina às quatro semanas de tratamento). ${ }^{3,7}$ Critérios de exclusão: idade inferior a 18 anos, antecedentes de gastrectomia/bypass gástrico, gestação em curso, perdas hemáticas conhecidas, anemia com outras causas além da ferropenia, alterações de outras linhagens celulares no hemograma (exceto trombocitose), ausência de estudo endoscópico digestivo alto e baixo (em homens e em mulheres pós-menopáusicas) e ausência de adesão à terapêutica com ferro oral.

Os dados foram obtidos através da consulta do processo clínico eletrónico (programa SClínico) constante do Centro Hospitalar Vila Nova de Gaia/Espinho, após aprovação do projeto pela Comissão de Ética deste centro hospitalar. Não foi realizado pedido de consentimento informado aos utentes incluídos no estudo. Dados foram recolhidos de forma anonimizada, através da atribuição de número de série a cada caso consultado, e registados neste formato em base de dados informática-Microsoft Excel 2016 ${ }^{\circledR}$.

Em cada doente foram consultados os registos da primeira consulta de hematologia, sendo a população estudada caracterizada quanto ao género, idade, origem do pedido de consulta (interno ou externo ao centro hospitalar do estudo), parâmetros analíticos (hemoglobina sérica, volume globular médio, ferritina), suplementação de ferro (dose de ferro elementar diário realizado, intervalo de tempo até repetição do hemograma e evolução do valor de hemoglobina) e verificada a ausência de algum dos critérios de exclusão definidos.

O rastreio da DC foi considerado se houvesse presença de anticorpos antiendomísio, antigliadina e/ou antitransglutaminase. O diagnóstico definitivo foi apenas considerado se existisse uma biópsia duodenal concordante.

Por sua vez, o rastreio da GAAI incluiu a pesquisa de anticorpo anticélula parietal e gastrina sérica. O diagnóstico definitivo foi considerado se existisse atrofia em biópsia gástrica.

Por último, o rastreio do Hp incluiu a realização de endoscopia digestiva alta (EDA) com biópsia e/ou teste respiratório com ureia marcada com o Carbono 13. Nos casos positivos foi também registada a terapêutica realizada para a erradicação, a confirmação da erra- 


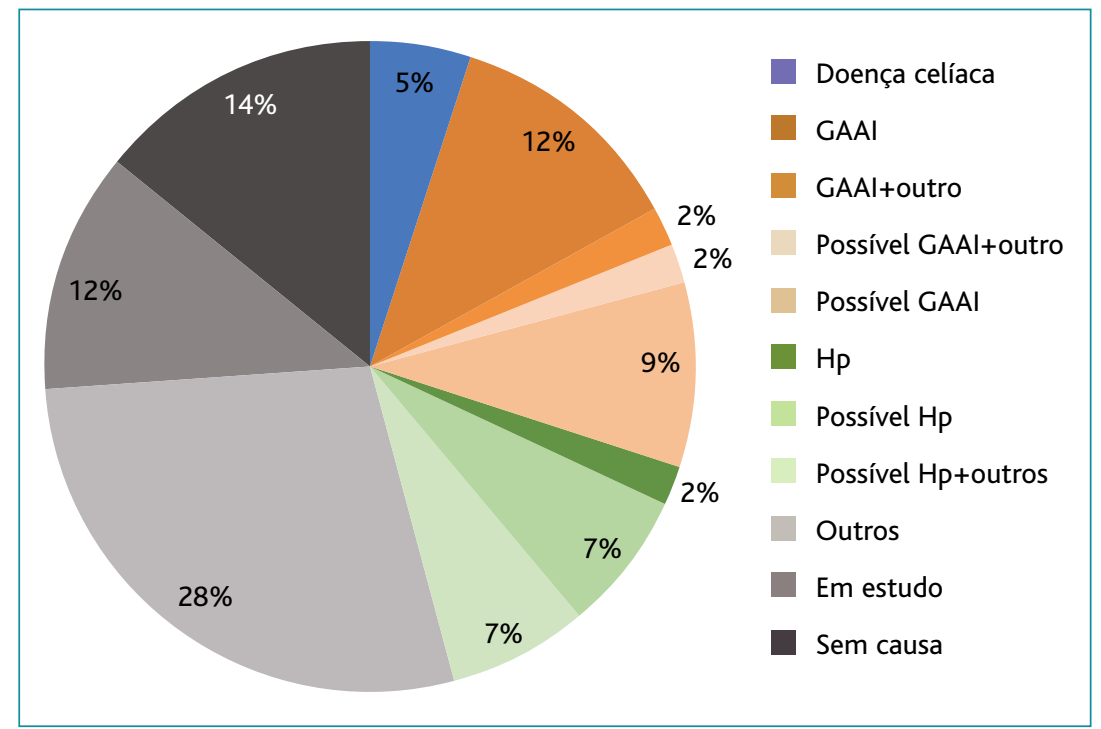

Figura 1. Causas identificadas da anemia na população em estudo que fez algum tipo de rastreio $(n=43)$.

Legenda: $\mathrm{GAAI}=$ Gastrite atrófica autoimune; $\mathrm{Hp}=$ Helicobacter pylori.

dicação (por EDA com biópsia ou teste respiratório da urease), bem como o efeito da mesma na anemia.

Todo o processo do estudo teve um parecer positivo da Comissão de Ética do Centro HospitalarVila Nova de Gaia/Espinho.

As variáveis categóricas são apresentadas como frequências e percentagem e as variáveis contínuas como média e desvio-padrão, ou mediana e amplitude interquartis para variáveis com distribuição não normal. $\mathrm{O}$ teste à normal distribuição dos dados foi realizado com recurso ao teste Shapiro-Wilk.

\section{RESULTADOS}

Durante o período em estudo foram realizadas 4.655 primeiras consultas no serviço de hematologia, das quais $518(11,1 \%)$ foram motivadas por anemia ferropénica. Após aplicação dos critérios de inclusão e exclusão identificaram-se 48 casos de ARF (aproximadamente 9,3\%) cujas etiologias principais estão representadas na Figura 1.

Os doentes inseridos no estudo apresentavam uma idade média de 51 anos (mínima de 18 anos e máxima de 84 anos), com predominância do sexo feminino $(93,8 \%)$. As medianas dos valores de hemoglobina e de ferritina foram de $10,25 \mathrm{~g} / \mathrm{dL}$ e $6 \mathrm{ng} / \mathrm{mL}$, respetivamente. A maioria dos pedidos de consulta $(60,4 \%)$ foi reali- zada pelos cuidados de saúde primários (Quadro I).

Dos 48 doentes incluídos no estudo metade efetuou rastreio completo com pesquisa das três patologias e 19 doentes realizaram para uma ou duas das entidades do estudo (rastreio parcial) (Figura 2). Nestes últimos não foi feito despiste de DC em sete casos, de GAAI em nove casos e de Hp em nove casos. Em cinco doentes não há registo de realização de nenhum dos rastreios.

Nos doentes em que foi efetuado o rastreio parcial para pelo menos uma das causas de má absorção de ferro em estudo (DC, GAAI ou Hp) ( $n=43$ ), este foi positivo em $62,8 \%$ dos doentes, subindo esse valor para $83,3 \%$ quando o rastreio foi completo, ou seja, realizado para as três patologias ( $n=24$ ) (Quadro II).

Entre aqueles que realizaram o rastreio para pelo menos uma das entidades, este foi positivo para DC em três doentes, mas apenas dois casos $(4,7 \%)$ foram con-

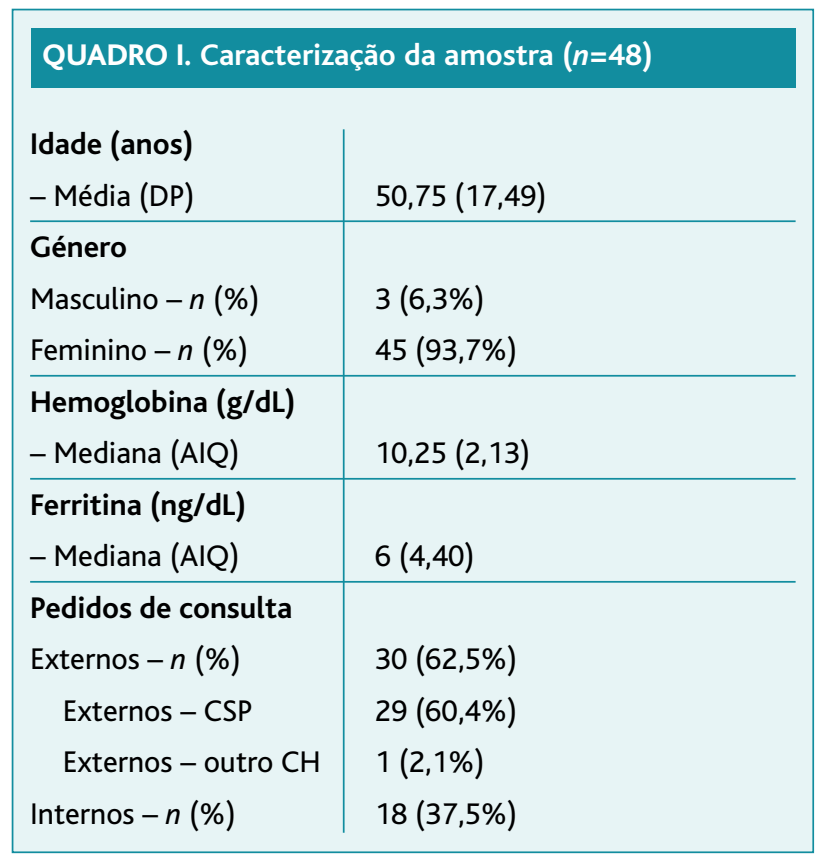

Legenda: CSP = Cuidados de saúde primários; $\mathrm{CH}=$ Centro hospitalar; $\mathrm{DP}=$ Desvio-padrão; $\mathrm{AIQ}=$ Amplitude interquartil. 
firmados após biópsia duodenal; para $\mathrm{Hp}$ foi positivo em 13 doentes (30,2\%); e para GAAI em 14 doentes, com seis casos (13,9\%) desta última doença confirmados após biópsia gástrica (em quatro casos houve perda de seguimento e num dos casos não foi pedida a biópsia). Em três doentes o rastreio foi positivo para GAAI e Hp concomitantemente.

Dos seis casos de GAAI confirmada por biópsia, o anticorpo anticélula parietal estava presente em todos os doentes e a gastrina sérica estava aumentada em quatro casos.

O efeito da erradicação do Hp sobre a anemia foi resolutivo em apenas um doente, nulo em seis doentes e desconhecido em seis casos, por ausência de hemograma após erradicação $(n=2)$, por ausência de registo de

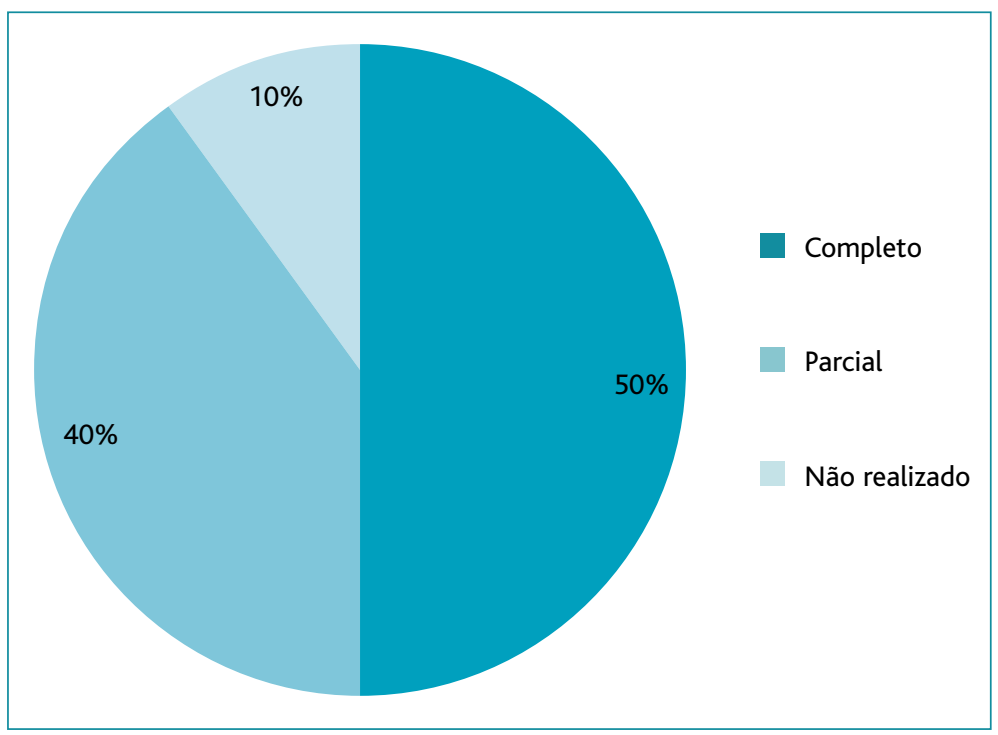

Figura 2. Tipo de rastreio realizado na população do estudo $(n=48)$.

Nos utentes que não fizeram rastreio de nenhuma das três entidades $(n=5)$, a etiologia não foi identificada $(n=4)$ ou permanece em estudo $(n=1)$.

De salientar que dos três doentes do sexo masculino incluídos no estudo, a etiologia da anemia foi atribuída a neoplasia do cólon $(n=2)$ e angiectasias do intestino delgado $(n=1)$.

Os restantes casos $(n=23)$ corresponderam a cinco doentes que ainda se encontravam a realizar o estudo etiológico da anemia, a seis doentes cuja anemia não tinha causa identificada e 12 doentes cuja anemia foi atribuída a outras etiologias, nomeadamente menorragia inicialmente não valorizada $(n=4)$, angiodisplasia do intestino delgado $(n=4)$, neoplasia do cólon $(n=3)$, doença de refluxo gastroesofágico/hérnia de hiato $(n=3)$, doença de Crohn ( $n=2)$, hemorróidas $(n=1)$ e polimorfismo genético que confere ferropenia $(n=1)$, havendo doentes com sobreposição destas patologias.

\begin{tabular}{|c|c|c|c|c|c|c|}
\hline \multirow{3}{*}{\multicolumn{2}{|c|}{$\begin{array}{l}\text { QUADRO II. AAvaliação } \\
\text { infeção por Helicobactel } \\
\text { oral }(n=48)\end{array}$}} & $\begin{array}{l}\text { s rastre } \\
\text { ylori, n }\end{array}$ & $\begin{array}{l}\text { para d } \\
\text { loentes }\end{array}$ & $\begin{array}{l}\text { a celía } \\
\text { n anem }\end{array}$ & $\begin{array}{l}\text { sastrite atrófica } \\
\text { erropénica refrat }\end{array}$ & $\begin{array}{l}\text { nune e } \\
\text { o ferro }\end{array}$ \\
\hline & & \multicolumn{5}{|c|}{ Resultado do rastreio } \\
\hline & & \multicolumn{4}{|c|}{ Rastreio positivo } & \multirow{2}{*}{$\begin{array}{l}\text { Rastreio } \\
\text { negativo } \\
n(\%)\end{array}$} \\
\hline $\begin{array}{l}\text { Tipo de rastreio } \\
\text { realizado }\end{array}$ & $\begin{array}{c}\text { Total } \\
(n)\end{array}$ & $\begin{array}{c}\mathrm{DC} \\
n(\%)\end{array}$ & $\begin{array}{l}\text { GAAI } \\
n(\%)\end{array}$ & $\begin{array}{c}\mathrm{Hp} \\
n(\%)\end{array}$ & $\begin{array}{l}\text { Total } \\
n(\%)\end{array}$ & \\
\hline Completo & 24 & 0 & $\begin{array}{c}11 \\
(45,8 \%)\end{array}$ & $\begin{array}{c}12 \\
(50 \%)\end{array}$ & $\begin{array}{c}20 \\
\text { (83,3\%; incluindo } 3 \\
\text { com GAAl e Hp) }\end{array}$ & $\begin{array}{l}4 \\
(16,7 \%)\end{array}$ \\
\hline Parcial & 19 & $\begin{array}{c}3 \\
(15,6 \%)\end{array}$ & $\begin{array}{c}3 \\
(15,6 \%)\end{array}$ & $\begin{array}{c}1 \\
(5,2 \%) \\
\end{array}$ & $\begin{array}{c}7 \\
(36,8 \%)\end{array}$ & $\begin{array}{l}12 \\
(63,2 \%)\end{array}$ \\
\hline $\begin{array}{l}\text { Completo } \\
+ \\
\text { Parcial }\end{array}$ & 43 & $\begin{array}{c}3 \\
(7 \%)\end{array}$ & $\begin{array}{c}14 \\
(32,6 \%)\end{array}$ & $\begin{array}{c}13 \\
(30,2 \%)\end{array}$ & $\begin{array}{c}27 \\
(62,8 \% ; \text { incluindo } 3 \\
\text { com GAAl e Hp) }\end{array}$ & $\begin{array}{l}16 \\
(37,2 \%)\end{array}$ \\
\hline Não realizado & 5 & - & - & - & - & - \\
\hline
\end{tabular}

Legenda: $\mathrm{DC}=$ Doença celíaca; $\mathrm{GAAI}=$ Gastrite atrófica autoimune; $\mathrm{Hp}=$ Helicobacter pylori . 


\section{DISCUSSÃO}

A DC, a GAAI e a infeção por Hp são etiologias reconhecidas da anemia ferropénica. Esta pode mesmo constituir-se como o único sinal de DC, ${ }^{3,8}$ estando desta forma recomendado pela Direção-Geral da Saúde o seu despiste em todos os casos de ferropenia. ${ }^{7}$ No caso da infeção por Hp, a relação causal não está tão bem estabelecida mas, de acordo com a evidência cumulativa atual, está recomendada a sua erradicação como parte do tratamento da anemia ferropénica inexplicada. ${ }^{3-4,8-9}$

Neste estudo, a realização do despiste destas entidades permitiu o diagnóstico de dois casos de DC, seis casos de GAAI e 13 doentes com infeção por Hp, equivalendo a uma prevalência de $4,7 \%$ para a DC, de cerca de $14 \%$ para a GAAI e de $30,2 \%$ para a infeção por Hp. Em comparação com alguns estudos, ${ }^{3,8}$ apenas a prevalência de DC é concordante (4-6\%), sendo a prevalência de GAAI e Hp na população do presente estudo inferior à reportada naqueles estudos (20-27\% e superior a $50 \%$, respetivamente). ${ }^{3,8}$

No entanto, dada a ausência de biópsia de confirmação da GAAI em cinco casos, uma eventual positividade a este nível subiria a respetiva prevalência no estudo para $25,6 \%$. Contudo, a prevalência pode também ser influenciada pelo tipo e origem da população inserida no estudo. De facto, num estudo efetuado no Egito em 25 doentes com ARF foi identificada DC em $44 \%$ e infeção pelo Hp em $48 \%$ dos doentes. ${ }^{9}$

Comparando a realização de rastreio completo com o rastreio parcial (de uma ou duas das entidades), verifica-se uma positividade superior em $20,5 \%$ no primeiro caso, o que reforça a importância da pesquisa das três entidades.

Ressalve-se ainda que a etiologia da anemia foi atribuída a outras condições clínicas em 27,9\% dos doentes que realizaram rastreio para pelo menos uma das entidades em estudo; isto aconteceu apesar de o rastreio ter sido positivo em alguns destes casos, o que chama a atenção para a multiplicidade de patologias que podem estar na origem da anemia ferropénica. Entre estes, quatro casos foram atribuídos a perdas menstruais ainda que no estudo tivessem sido excluídas as doentes com menorragia na primeira consulta. Este ponto realça a importância de uma anamnese cuidada, bem como a dificuldade da avaliação dos ciclos menstruais, dada a subjetividade individual na carac- terização da intensidade das perdas menstruais e da impossibilidade prática da sua quantificação objetiva.

Este estudo tem como limitações o tamanho da amostra, a impossibilidade de avaliar a resposta à erradicação do Hp em seis dos 13 casos identificados, não ter sido considerada a qualidade da prescrição antibiótica e adesão terapêutica e o facto de ter sido apenas avaliada a positividade ou negatividade dos marcadores séricos, sem ter em consideração o seu grau de elevação em comparação com os valores de referência. Por outro lado, existem outras causas, nomeadamente genéticas, ${ }^{10}$ que não foram abordadas neste estudo.

Apesar das limitações referidas, este estudo permite avaliar a influência da DC, GAAI e infeção por Hp na ARF em Portugal.

Este estudo reforça a importância do despiste da DC, GAAI e infeção por Hp nos casos de ARF, sendo ainda necessários mais estudos a este nível.

É também de salientar a importância da elaboração de protocolos de atuação que uniformizem a abordagem dos doentes com anemia ferropénica refratária ao ferro oral, o que possibilitará a redução do número de casos suspeitos de GAAI aos quais não foi realizada biópsia de confirmação e permitirá um follow-up adequado dos doentes com infeção por Hp, de forma a assegurar a avaliação do efeito da erradicação da infeção na anemia.

Ao nível dos cuidados de saúde primários é possível apenas efetuar este despiste de forma parcial, através do doseamento dos anticorpos anti-transglutaminase e anticélulas parietais gástricas e da realização de biópsia gástrica para despiste de GAAI e Hp, aquando da realização de endoscopia digestiva alta para exclusão de outras condições clínicas.

Em suma, a abordagem da anemia ferropénica refratária ao ferro oral permanece um desafio na prática clínica, sendo a colaboração e abordagem multidisciplinar uma mais-valia.

\section{REFERÊNCIAS BIBLIOGRÁFICAS}

1. Kassebaum NJ, Jasrasaria R, Naghavi M, Wulf SK, Johns N, Lozano R, et al. A systematic analysis of global anemia burden from 1990 to 2010 . Blood. 2014;123(5):615-24.

2. Short MW, Domagalski JE. Iron deficiency anemia: evaluation and management. Am Fam Physician. 2013;87(2):98-104.

3. Hershko C, Camaschella C. How I treat unexplained refractory iron deficiency anemia. Blood. 2014;123(3):326-33. 
4. Camaschella C. Iron deficiency: new insights into diagnosis and treatment. Hematology Am Soc Hematol Educ Program. 2015;2015:8-13.

5. Okam MM, Koch TA, Tran MH. Iron supplementation, response in irondeficiency anemia: analysis of five trials. Am J Med. 2017;130(8):991.e1991.e8.

6. Fonseca C, Marques F, Robalo Nunes A, Belo A, Brilhante D, Cortez J. Prevalence of anaemia and iron deficiency in Portugal: the EMPIRE study. Intern Med J. 2016;46(4):470-8.

7. Direção-Geral da Saúde. Abordagem, diagnóstico e tratamento da ferropénia no adulto: norma n. ${ }^{\circ}$ 030/2013, de 31/12/2013, atualizada em 09/04/2015. Lisboa: DGS; 2015.

8. Hershko C, Hoffbrand AV, Keret D, Souroujon M, Maschler I, Monselise $Y$, et al. Role of autoimmune gastritis, Helicobacter pylori and celiac disease in refractory or unexplained iron deficiency anemia. Haematologica. 2005;90(5):585-95.

9. Fayed SB, Aref MI, Fathy HM, Abd El Dayem SM, Emara NA, Maklof A, et al. Prevalence of celiac disease, Helicobacter pylori and gastroesop- hageal reflux in patients with refractory iron deficiency anemia. J Trop Pediatr. 2008;54(1):43-53.

10. Raposo F, Melo T, Costa M, Pereira M, Cleto E, Costa E, et al. Anemia ferropriva refratária ao ferro: uma entidade clínica de descrição e caracterização molecular recentes [Iron-refractory iron deficiency anaemia: a clinical entity with recent molecular characterisation and description]. Acta Pediatr Port. 2015;46(4):401-5. Portuguese

\section{CONFLITO DE INTERESSES}

Os autores declaram não ter quaisquer conflitos de interesse.

\section{ENDEREÇO PARA CORRESPONDÊNCIA}

Ana Carolina Teixeira

E-mail: t.carolina@hotmail.com

http://orcid.org/0000-0003-2078-7923

Recebido em 27-12-2017

Aceite para publicação em 05-02-2019

\section{ABSTRACT}

\section{CELIAC DISEASE, AUTOIMMUNE ATROPHIC GASTRITIS AND HELICOBACTER PYLORI IN ORAL IRON THERAPY RESISTANCE ANEMIA}

Objectives: Identify the number of celiac disease (CD), autoimmune atrophic gastritis (AAG) and the Helicobacter pylori ( $\mathrm{HP}$ ) infection in patients with iron-refractory iron deficiency anemia (RIA).

Type of study: Observational, retrospective, longitudinal.

Location: Hospital center hematology unit in the north region of Portugal.

Population: All first hematology consultations of patients with RIA between 2011 and 2016.

Methods: Collection of data using the electronic clinical file (socio-demographic, origin of the consultation request and diagnostic tests data). Microsoft Excel $2016^{\circledR}$ was used to analyze and process the data.

Results: We identified 48 cases of iron-refractory iron deficiency anemia (9.3\% of 518 patients with iron deficiency anemia). A complete screening for $C D, A A G$ and the infection by $\mathrm{Hp}$ was made for 24 patients. A partial screening (only for one or two of these diseases) was made for 19 patients. In the 43 cases that at least one of the screenings were made, the prevalence of CD was about $4.7 \%$, the prevalence of AAG was $14 \%$ and the infection by $\mathrm{Hp}$ was $30.2 \%$. We couldn't evaluate the Hp eradication because it wasn't confirmed in six of 13 cases identified with this pathology. In the other seven cases, Hp eradication solved anemia in only one patient.

Conclusion: We identified a high percentage of patients with iron-refractory iron deficiency anemia that suffer from AAG and infection by $\mathrm{Hp}$, as is referred to in international studies. These findings reinforce the role of these diseases in iron-refractory iron deficiency anemia in the Portuguese population. This work reinforces the need to screen for CD, AAG and Hp infection in iron-refractory iron deficiency anemia.

Keywords: Anemia, iron-deficiency; Celiac disease; Gastritis, atrophic; Helicobacter pylori; Iron-refractory; Iron deficiency anemia. 